\title{
An fMRI Study of Bilingual Sentence Comprehension and Workload
}

\author{
Mihoko Hasegawa,*'† Patricia A. Carpenter,* and Marcel Adam J ust* \\ *Center for Cognitive Brain Imaging, Department of Psychology, Carnegie Mellon University, Pittsburgh, Pennsylvania 15213; and \\ †Graduate School of Science and Technology, Chiba University, Chiba, J apan
}

Received May 15, 2001; published online J anuary 22, 2002

To examine the relation between the cortical substrates that support the comprehension of one's native language and those that support a second language, fMRI measures of cortical activation were taken as native J apanese participants, who had acquired moderate fluency in English, listened to auditory sentences in J apanese and English. In addition, to examine the impact of processing difficulty within a language, sentence difficulty was manipulated by including affirmative (easy) and negative (hard) sentences. The volume of activation was greater for English in most of the cortical regions, suggesting that more cognitive effort was required to process English. Also, a high percentage of the voxels that were activated for the J apanese condition were also activated for the English condition, with as much overlap between J apanese and English as between the processing of affirmative and negative sentences within J apanese. Negative sentences elicited greater activation than affirmative sentences primarily for English, indicating that the structural difficulty of negation has a larger impact on cortical activation if it occurs in the context of the second language, which may serve as another source of difficulty. These results suggest that a shared network of cortical regions supports the processing of both a first and a second language, such that the second language requires more computation and activity from the network. 02002 Elsevier Science (USA)

\section{INTRODUCTION}

The issue of how two languages are implemented in a bilingual's brain has been addressed by neuropsychological studies of bilinguals who have suffered language impairments due to brain damage (e.g., Paradis, 1997; Vaid, 1980). However, the advent of neuroimaging enables researchers to assess issues of language representation in bilinguals more directly. This study uses functional magnetic resonance imaging (fMRI) to examine the processing of auditorily presented sentences in two languages from different language families, J apanese and English, by J apanese native speakers who learned English relatively late (during adolescence). In addition to examining the issue of the relative cortical localizations of the two languages, we also examine bilingual language processing from the vantage of a workload perspective. This was done by varying the difficulty of the comprehension task by including both easier (affirmative) and harder (negative) sentences in each language. These two perspectives mutually illuminate how the patterns of activation associated with these two languages may reflect on the cortical processing of a first and second language (L1 and L 2).

Previous neuroimaging studies primarily have investigated the issue of bilingual language processing from a perspective of localization, by examining the cortical activation loci subserving $L 1$ and $L 2$ processes. Such studies have differed along many dimensions that would seem potentially important. One such variable is the relative fluency of the participants, which often also correlates with the age of acquisition of the second language (Perani et al ., 1998; Wartenburgher et al., 2001). Another variable is the similarity of the two languages, for example, whether they are as different as J apanese and English, as in the current study, or as similar as Spanish and Catalan. ${ }^{1}$ The implicit hypothesis is that similar languages may be more likely to draw on overlapping cortical systems than dissimilar languages (Vaid, 1983).

Another source of variation among neuroimaging studies of bilingualism arises from the tasks that are used, typically involving either production or comprehension. Additionally, even within comprehension, some studies utilized auditory comprehension while others have probed reading, and some studies have involved only single words while others have involved larger units, including whole stories. The impact of these variables on the cortical representation of $L 2$ is difficult to assess, given that so few of these issues have been examined systematically. Nevertheless, we will

\footnotetext{
${ }^{1}$ Spanish and Catalan are both members of the Indo-E uropean family, while English and J apanese are from different language families; English is a member of the Indo-European family, and $\mathrm{J}$ apanese is considered to be isolated or a member of the Altaic family (Cristal, 1998).
} 
briefly describe some of these findings, as well as situate the current study among these precedents, before turning to the specific rationale that motivates it.

For highly fluent bilinguals who acquired their second language early, a number of comprehension studies have found evidence that there is extensive overlap for the two languages in the classical language areas, i.e., roughly Wernicke's area and Broca's area. One study that resembles the present one involved sentence comprehension of two dissimilar languages, Mandarin and English, ${ }^{2}$ although the material was presented visually rather than auditorily (Chee et al., 1999a); it found highly overlapping activation in the left frontal and temporal language areas for L1 and L2. As for auditory processing, a study that involved listening to stories also found considerable overlap in the left temporal regions and hippocampal structures for one's native language and a second language, Spanish and Catalan, two similar languages of the Indo-European family (Perani et al., 1998).

Studies that involved the generation of words also found overlap in areas of the left frontal, temporal, and parietal regions for highly fluent bilinguals (Chee et al., 1999b; Hernandez et al., 2000, 2001; Kim et al., 1997; Klein et al., 1999), even though they varied in the combination of languages. These results suggest that bilinguals' two languages may be represented in common cortical regions irrespective of the language similarity, at least for highly fluent bilinguals who acquired their languages early. One contrasting result, obtained in a task that required speech output, found that the second language elicited additional activation in the left putamen, which is thought to be due to motor control, although there was no difference in group-averaged activation for French and English in the frontal language region (Klein et al., 1994, 1995).

In contrast to the early-acquired, highly fluent bilinguals, for the highly fluent bilinguals who acquired L2 relatively late, Kim et al. found a great degree of overlap in Wernicke's area, but considerable spatial separation with less overlap of the two languages in B roca's area relative to the results of those who acquired their languages early, as described above (Kim et al., 1997). However, a study that also examined late-acquired, highly fluent bilinguals revealed common activation in Broca's area in a task that involved comprehension of visually presented words (Illes et al., 1999). These contradicting results suggest possible distinctions among areas that are often coactivated in language tasks, perhaps as a function of the tasks.

Although the amount of activation for L1 and L2 typically has not been the focus of most investigations, there appears to be some divergence in the findings,

\footnotetext{
${ }^{2}$ Chinese is a member of the Sino-Tibetan language family (Cristal, 1998).
}

particularly from studies with moderately fluent bilinguals. In a task involving listening to dialogues, Nakai et al. (1999) reported more activation for $L 2$ than for $L 1$ in most of the regions of interest, especially in Broca's area, the angular gyrus, the middle frontal gyrus, and the supplementary motor area. Chee et al. (2001) reported a robust activation in the bilateral opercular region for only $L 2$ with their less proficient bilingual group, which they attributed to the greater workload to process L2. By contrast, in a similar task involving listening to short stories, Perani et al. (1996) reported a smaller range of activation for $L 2$ than for $L 1$, especially in the left temporal region. In another story listening task, Dehaene et al. (1997) also found a trend of less activation for L2 in the left temporal region as well as a high interparticipant variability for $L 2$, varying from complete right lateralization to the standard left lateralization, including disappearance of activation from Wernicke's area for the second language.

In considering these results, it might be hel pful to explicitly consider the task from the perspective of the cognitive workload that the task imposes on the listener. For example, in a passive listening task, one important factor may be whether the participants' comprehension levels are comparable in the two languages. Some decrement in activation for L 2 may occur because of a lower level of comprehension. Thus, it may be important to assess behavioral performance that gives an estimate of comprehension level along with the degree of activation. In the current study we test whether the comprehension level is systematically related to the amount of activation in the language processing regions.

The current study applies the perspective of cognitive workload by considering L2 comprehension as a potentially more difficult task than LI comprehension, as well as considering how affirmative and negative sentences within L2 may also differ in terms of workload. The workload perspective grows out of a number of recent brain-imaging studies in a variety of domains that have indicated that within some range, the amount of brain activation is affected by the quantitative computational demand imposed by a cognitive process. This result has been found in word span tasks as the span increases (Grasby et al., 1994), in n-back working memory tasks as $\mathrm{n}$ increases (Braver et al., 1997; Rypma et al., 1999), in mental rotation as the degree of rotation increases (Carpenter et al., 1999a), and in auditory sensory tasks as the rate of stimulus presentation increases (Price et al., 1992). For example, in a sentence comprehension task, the amount of activation in a network of areas increased as a function of the complexity of the sentence (J ust et al., 1996). That study contrasted three types of two-clause sentences that varied in their structural complexity from relatively easy conjoined active sentences, to moderately difficult sentences with embedded subject-rela- 
tive clauses, and to the most complex sentences with embedded object-relative clauses. Although sentence length and lexical content were equivalent, the results showed that fMRI-measured activation increased with sentence complexity in both of the classic left-hemisphere language areas and to a much lesser extent in their right homologues. A related study manipulated not only the syntactic complexity of the sentence, but also the frequency of the nouns (Keller et al., 2001). The two sources of complexity, syntactic and lexical, led to overadditive interactions in the amount of activation, such that the effect of the two sources of difficulty was much greater than the effect of either one alone in several regions, including the classic left hemisphere regions associated with language processing. Such studies support the view that the allocation of cognitive effort is reflected by fMRI-measured activation and affected, in part, by task difficulty.

In the current study the processing in a nonnative language is one source of difficulty, by virtue of the participants' lower fluency in L2 than L1. The workload perspective leads to the hypothesis that native J apanese speakers' comprehension of English would elicit more brain activation than comprehension of J apanese, at least if participants attempted to understand the sentences to the same level in the two languages. As a second source of difficulty, we manipulated sentence complexity by comparing affirmative and negative sentences, such as The man picked up a key and gavea box to the girl versus The doctor wrotea card and didn't bring a toy for the child. Negation is an interesting manipulation in the context of bilingualism, in part because it exists in all languages and is marked as more complex than the affirmative. In addition, numerous behavioral studies in English indicate that negative sentences are behaviorally more difficult to process than affirmatives, resulting in increased reading time and errors (Carpenter and J ust, 1975; Chase and Clark, 1972). The modal-processing model for negation suggests that negative sentences are encoded as an affirmative core with an additional linguistic marker signaling the negation. For example, the sentence "The address isn't an even number" might be coded initially like "That the address is an even number isn't true." Thus, the comprehender may initially process the affirmative core (the address is an even number) and then must reverse the polarity of the representation ((the address is an even number) is false) to accommodate the negation; this reversing contributes to the increase in processing time and errors for negative compared to affirmative sentences.

That very complex negative sentences can lead to more cortical activation in one's native language was supported by an imaging study of (monolingual) English reading that used sentences with a complex negative embedding clause (It isn't true that the star is above/below the plus) (Carpenter et al., 1999b). That study used high-speed echoplanar fMRI to trace the time course of the $\mathrm{MRI}$ R-measured activation in a small number of cortical areas as participants comprehended single sentences. In three areas, the left posterior temporal area and the left and the right parietal regions, there was increased activation for negative sentences compared to affirmative sentences. The current study used simpler negative sentences in a blocked design, features which were intended to decrease the errors associated with processing the whole sentence.

Another point of the rationale underlying the current study is the emerging view that language processing is implemented in a network of areas that may be differentially affected by particular processes, but which are not necessarily affected solely by one level of a linguistic variable (such as lexical complexity or structural complexity). If the activation associated with sentence comprehension is examined relative to a baseline task that involves none of the component processes, activation is observed in numerous cortical sites that appear to collaborate in comprehension. These include areas that have been classically associated with language processing based on neuropsychological evidence, including the left posterior/middle temporal region, the left inferior frontal gyrus, and the angular gyrus (Bavalier et al., 1997; Binder et al., 1997; J ust et al., 1996; Keller et al., 2001). The areas that have been associated with articulation, the left precentral region and the supplementary motor area (Smith and J onides, 1998a), or with phonological retention, the left supramarginal gyrus (Smith and J onides, 1998b), also showed activation in tasks of comprehension compared to resting or nonlinguistic baseline conditions (Binder et al., 1997; Keller et al., 2001; Michael et al., 2001; Nakai et al., 1999). Although the left precentral sulcus region is typically associated with spatial attention and eye movement, activation in this region during auditory sentence comprehension (Binder et al., 1997; Michael et al., 2001; Nakai et al., 1999) suggests that eye movements or spatial attention is not a necessary condition for its activation and that this region is also implicated in some aspect of language comprehension. Thus, the cortical system is dynamic and can recruit other regions, both proximally and distally, depending in part on the task. Consequently, it is potentially informative to examine how the language comprehension network system responds to difficulty in the two languages of a bilingual.

Although there are numerous hypotheses concerning the processes implemented in particular cortical regions, it is also possible that processes may not be so discretely localized. In fact, studies that attempt to map particular processes onto single cortical regions, in order to localize the orthographic, phonological, and semantic components of language, often yield conflicting conclusions (see Keller et al., 2001). Such mappings are often quite sensitive to the properties of the two 
particular tasks being compared, reflecting strong limitations in the subtractive logic that typically underlies such comparisons. But beyond limitations of the methodology, the evidence suggests that language processing is accomplished by large-scale, variable, and distributed patterns of activity among cooperating and interactive cortical areas rather than in modules that serially and discretely perform single processes.

Taking the emerging view of a distributed cortical network for language processing, together with the perspective of cognitive workload, we compare the activation engendered by the many processes that constitute the comprehension of affirmative and negative sentences in $L 1$ and $L 2$, including encoding the stimuli, constructing a representation of the sentence's structure and meaning, and phonological rehearsal or retention in order to answer a subsequent probe. The data analyses examine how the volume of activation in a set of a priori defined regions is modulated for the two languages and the two types of sentences, to provide a better understanding of how the second language is cortically represented relative to the native language.

\section{MATERIALS AND METHODS}

\section{Participants}

Ten right-handed native J apanese speakers gave signed, informed consent to participate in this study approved by the University of Pittsburgh and Carnegie Mellon Institutional Review boards. All but one of the participants had learned English as their second Ianguage after the age of 12 and had moved to the United States sometime after completing college level education in J apan; the exception had moved to the United States at the age of 15 after completing junior high school in J apan ( $\mathrm{M}=26.9, \mathrm{SD}=4.8$, range $21-38$ ).

Each participant completed a language background questionnaire that involved self-ratings of their listening fluency in English on a scale of 1.0-5.0 with 0.5 intervals, where 5 was excellent. Eight participants rated themselves between 2.5 and 3.5, except for two; one who assigned herself a rating of 5 and one who assigned $1.5(\mathrm{M}=3.0, \mathrm{SD}=0.9)$.

\section{Stimulus Materials}

The participant's task was to listen to a target sentence, followed $1 \mathrm{~s}$ later by a probe sentence, and then to press one of two buttons to indicate whether the probe was true or false relative to the target. All of the target sentences had two clauses, such as The worker read a magazine and showed some pictures to the brother. Half of the target sentences were affirmative and half were negative. Probe sentences had one clause, consisting of the words used in the preceding target, such as The worker read a magazine (true) or
The brother read a magazine (false). Negative sentences contained a negated verb in either the first or the second clause, such as The doctor read a book and didn't bring the baggage to the visitor, followed by a probe such as The doctor didn't bring the baggage (true) or The visitor read a book (false). Fifty-five percent of the probes were true. The nouns and verbs for English sentences were chosen to be at the level of junior high school, namely beginner or lower intermediate levels, and therefore would be familiar to the participants. Translation equivalents were used in the twolanguages to make sure that the lexical level would be comparable, although the combination of verbs and nouns differed, and consequently, the sentences were comparable but not identical. The stimulus sentences and probes were recorded digitally by native speakers and presented over headphones. The speed of the sentences was slightly slower than normal for either language, and both the duration of the sentences and the clarity were equated as much as possible both within and across the speakers (the mean duration was 4371 ms and 4754 ms, for English and J apanese, respectiveIy). In an experimental debriefing, all the participants described both the clarity and the speed of the stimuli in the two languages as appropriate.

A response time for each trial was measured from the onset of the probe sentence; however, we report times in which we have subtracted off the average probe duration of each condition (mean $=2439$ and $2797 \mathrm{~ms}$, for English and J apanese, respectively) to equate for the slight difference between languages. In addition, we recorded the accuracy of the response.

Three English-speaking bilingual controls who were comparably fluent in J apanese (by self-rating) showed that the English sentences were quite comprehensible to native English speakers. Their response times and error rates were 1164 (2\%) and 1214 ms (2\%) for the affirmative and negative conditions, respectively. By contrast, their comparable response times and error rates in J apanese were 1394 (10\%) and 1745 ms (19\%). These behavioral results suggest that the effects to be reported are not specific to English and J apanese, but rather to their roles as representing $L 1$ and $L 2$.

\section{Procedure}

The presentation order of the J apanese and English conditions was balanced across participants. Each English or J apanese condition consisted of eight epochs, and each epoch had a series of five successive affirmative or negative trials (referred to as the polarity variable). Each trial consisted of a set of one target and one probe sentence, which were separated by a 1000-ms pause, and was terminated by the participant's key response. A 1500-ms pause followed the response before the onset of the next trial. Sentence epochs alternated with fixation periods, which were alternations of 
6 and $30 \mathrm{~s}$. The 30-s fixation periods constituted the baseline, and five of them were interspersed throughout the experiment. Six-second fixation periods intervened between successive sentence epochs and were included to allow the hemodynamic response to decrease before a different type of sentence epoch began.

The participants were given practice with the sentence verification task before the imaging study through instructions and participation in a short practice study. They were also familiarized with the scanner and the scanning procedure before the actual study began.

\section{Image Acquisition}

Images were acquired on a 1.5-T scanner (with quadrature birdcage head coil) at the MR Research Center at the University of Pittsburgh Medical Center. The functional images were obtained by using blood oxygenation level-dependent contrast (Ogawa et al., 1990). The acquisition parameters for gradient-echo EPI with 14 oblique axial slices were TR $=3000 \mathrm{~ms}$, $\mathrm{TE}=50 \mathrm{~ms}$, flip angle $90^{\circ}, 128 \times 64$ acquisition matrix, FOV $40 \times 20 \mathrm{~cm}$, 5-mm slice thickness, 1-mm gap, in-plane voxel resolution of $3.125 \times 3.125 \mathrm{~mm}$, and RF whole-head coil. The structural images were acquired in the same session for anatomical reference for cortical parcellation, using high-resolution T1-weighted spin echo, with TR $=400 \mathrm{~ms}$, TE $=11 \mathrm{~ms}, 256 \times 256$ acquisition matrix, 5-mm thickness, and 1-mm gap.

Image preprocessing of the functional data, including correction for in-plane motion and signal drift, was performed using FIASCO (Eddy et al., 1996). The maximum permissible mean of the in-plane estimated displacement was set to 0.2 voxels, and the mean across participants was less than 0.1 voxel. One participant showed large amounts of head motion in the experimental conditions and those data were omitted from the analysis; the data from the remaining nine participants were used. Data from the $6 \mathrm{~s}$ of fixation between epochs and from the first $6 \mathrm{~s}$ of each epoch were discarded to accommodate the rise of the hemodynamic response (Bandettini et al., 1992).

A voxel was considered active if a paired-sample $t$ test comparing its voxel-wise activation in a task condition to the fixation baseline reached a threshold value of $\mathrm{t}>4.5$, a high threshold that helped to compensate for the multiple comparisons. To reduce the potential influence of large vessels, voxels showing a signal change (relative to the fixation baseline) greater than $6.2 \%$ were excluded from all subsequent analyses. $^{3}$

\footnotetext{
${ }^{3}$ The threshold was set to separate the distribution of signal change by cortical function, which normally ranged $1-4 \%$, from that by vascular artifacts, which ranged $10-20 \%$.
}

\section{Anatomical Regions of Interest (ROI) and Data Analysis}

To compare the amount of activation in a given area across experimental conditions, anatomically defined ROIs were drawn for each participant using modification of the parcellation scheme described by Rademacher et al., (1992) and Caviness et al., (1996). This method uses limiting sulci and anatomically landmarked coronal planes to segment cortical regions. The anatomical information in the structural images was displayed in the three orthogonal planes simultaneously and the ROIs were manually drawn on each functional slice by M.H., after extensive training on the Rademacher/Caviness parcellation scheme. ${ }^{4}$

ROI s were drawn for areas that have been associated with reliable activation in related studies of language processing and their right hemisphere homologues. The temporal ROI, roughly corresponding to Wernicke's area, included the superior temporal gyrus (T1a, T1p; BA 22) and the middle temporal gyrus regions (T2a, T2p, and TO2; BA 21 and 37). The Broca's area counterpart consisted of a pars opercularis ROI, which is the posterior portion of the inferior frontal gyrus (F 30; BA 44), and a pars triangularis ROI, which is the anterior portion of the inferior frontal gyrus (F 3t; BA 45, and 47). The two parietal ROIs were the angular gyrus (AG; BA 40) and the supramarginal gyrus (SGa; BA 39). The left precentral sulcus ROI included the superior portion of the precentral sulcus and its posterior branches (FEF and PRG; BA 6), and the supplementary motor ROI corresponded to the juxtaparacentral lobule (J PL; BA 4). The auditory sensory areas were defined as the bilateral Heschl's gyrus ROIs (H1; BA 41 and 42).

To quantify the amount of activation in the four conditions ( apanese and English for affirmative and negative sentences), we computed two related dependent measures. The main measure was the number of significantly activated voxels in an ROI for each condition. The second measure was the average percentage signal change relative to the average fixation baseline for any voxel that reached threshold in that ROI for either of the two polarity conditions in that language. We draw notice to the fact that the signal change measure seldom reached statistical signifi-

\footnotetext{
${ }^{4}$ The interrater reliability of this ROI-defining procedure between two trained staff members was evaluated for 4 of the 18 ROIs in two participants in a comparable study. The reliability measure was obtained by dividing the size of the set of voxels that overlapped between the two raters by the mean of their two set sizes. The resulting eight reliability measures were in the $78-91 \%$ range, with a mean of $84 \%$, as high as the reliability reported by the developers of the parcellation scheme. For each participant, a mean of the functional images was registered to the high-resolution, T1-weighted structural volume scan image and placed in parallel alignment with the anterior commissure-posterior commissure line. The functional images were then segmented in the axial plane by manually delineating the ROIs on each functional slice.
} 


\section{TABLE 1}

The Percentages of Voxels Activated in J apanese That Had an Identical or Adjacent Voxel Activated in English

\begin{tabular}{lcr}
\hline \multicolumn{1}{c}{ ROI } & Identical & $\begin{array}{r}\text { Adjacent/ } \\
\text { identical }\end{array}$ \\
\hline Left temporal & $51 \%$ & $83 \%$ \\
Left angular gyrus & $27 \%$ & $84 \%$ \\
Left precentral sulcus & $57 \%$ & $80 \%$ \\
Left Heschl's gyrus & $74 \%$ & $100 \%$ \\
Right Heschl's gyrus & $50 \%$ & $69 \%$ \\
\hline
\end{tabular}

cance, perhaps because of the smaller number of observations. In other words, subjects with no significantly activated voxels in either J apanese or English for a ROI were not included in this signal intensity analysis, while they were included in the voxel count analysis, given a value "zero." N everthel ess, the effects for the two measures were always in the same direction. For each measure for each ROI, language and polarity effects were statistically treated as withinsubject factors using a two-way ANOVA. Then, additional tests for the simple main effect of polarity were carried out separately for each language.

To assess the overlap among activated voxels, we calculated two proportions for each ROI, focusing on those regions with the largest number of activated voxels. The first, called "identical voxels," was the proportion of voxels activated in both language conditions compared to the total number of activated voxels in J apanese, the participants' native language. Participants who had no voxels activated in one condition were not included (which affected the calculations for the left supramarginal gyrus and the left opercular regions). The second and more lenient criterion counted voxels as overlapping if a voxel activated in the J apanese condition was adjacent to a voxel activated in English; adjacent was defined in terms of a voxel's center being less than $6 \mathrm{~mm}$ from the center of the activated voxel.

\section{RESULTS AND DISCUSSION}

\section{fMRI Results}

\section{Location Overlap}

The location of the activation was similar in the two languages, as indicated by the considerable overlap in activated voxels. This value, shown in Table 1 , was quantified by computing overlap on a voxel basis for five ROIs that had the most activation, namely, the left temporal, the left angular gyrus, and the left precentral sulcus ROIs and the bilateral Heschl's gyri. To evaluate how this degree of overlap compares to the overlap of activated voxels within a language, we calculated for each participant the percentage of activated voxels in the left temporal region for the J apanese affirmative condition that were identical or were adjacent or identical to voxels that were activated for the negative condition. The average percentages were $62 \%$ for identical voxels and $77 \%$ for adjacent or identical voxels, percentages that may reflect differences in the two sentence conditions within a language as well as the inherent variance in the neural processing of sentences and its measurement with fMRI. The $62 \%$ overlap within the two J apanese language conditions can be compared to $51 \%$ identity overlap in the left temporal across English and J apanese; the $62 \%$ is a type of upper bound on the amount of overlap; it indicates that the between-language overlap is extremely high, $82 \%$ of the maximum possible.

This high degree of location overlap contrasts with the finding of less overlap in Broca's area between the two languages for bilinguals who were performing internal speech tasks (Kim et al., 1997). Of course, the task reported by Kim et al. involved production rather than comprehension and, hence, a difference in task as well as area. In the current study, the amount of activation in the left inferior frontal regions, particularly pars opercularis, was relatively low and so constituted a weak test of the hypothesis; nevertheless, the overlap was $54 \%$ for identical voxels and $84 \%$ for adjacent or identical voxels, indicating considerable overlap for this region as well. This suggests that the choice of region of interest is not the source of the discrepancy in findings, and future studies may find it interesting to compare voxel overlap for production and comprehension tasks directly.

This analysis of overlap in the cortical processing of English and J apanese sentences is limited, of course, by the spatial resolution of the technology. It is theoretically possible that the networks that are reflected in the activation are not overlapping, but only spatially adjacent within the $3.25 \times 3.25 \times 5-\mathrm{mm}$ voxels in which the data were acquired. This constraint aside, it is neverthel ess informative that the overlap of the neural substrate underlying the comprehension in the two languages approaches the overlap observed between two conditions within one's native language. Although we will present a quantitative analysis that supports this hypothesis, Fig. 1 illustrates the results by presenting functional activation images superimposed on structural images for several slices for one representative individual. The mean activation for the English (negative) condition (in the top row) is much higher than that for the J apanese (negative) condition (in the bottom row) for several anatomical regions.

\section{Total Activation}

The perspective of differential cognitive workload in $L 1$ and L2 suggests that the amount of activation associated with the four conditions might vary systemat- 


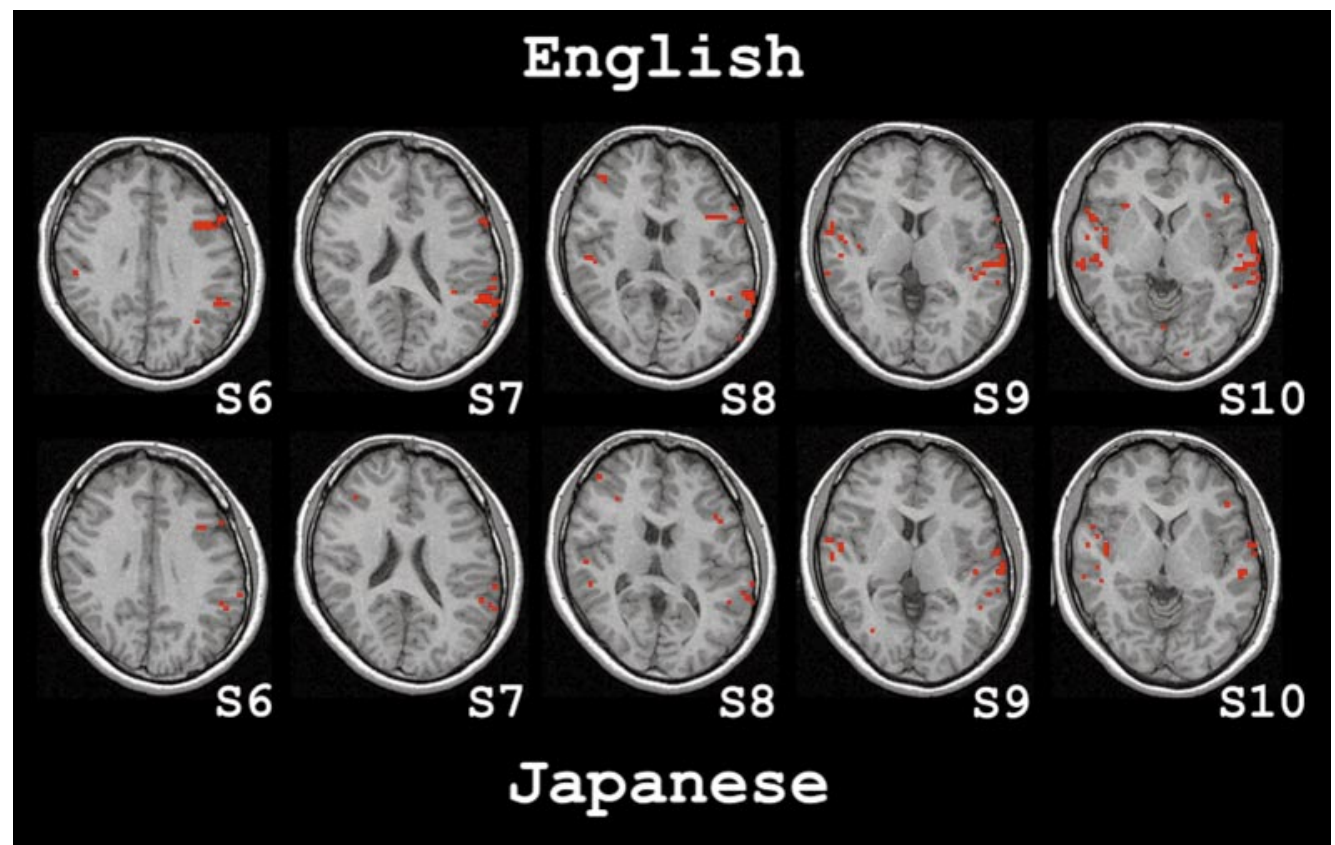

FIG. 1. Thresholded fMRI brain activation images (superimposed on structural images) showing the greater number of activated voxels in the English (negative) condition (in the top row) compared to the J apanese (negative) condition (in the bottom row) in five successive slices for a typical participant. (Following radiological convention, the images are left-right reversed.) Slice S6 shows activation in the left precentral sulcus and, more posteriorally, in the angular gyrus region. Slice S7 also shows considerable activation in the left angular gyrus region. Slice S8 shows more anterior activation in the left pars opercularis and left angular gyrus and in the right pars triangularis and right temporal regions. Slice S9 shows activation in the right and left Heschl's regions, as well as the left temporal region. Slice S10 shows activation mostly in the right Heschl's and the left and right temporal regions; the more anterior activation is in the inferior frontal region, primarily the pars triangularis.

ically. We first discuss the total activation summed over the relevant ROI s, shown in Figs. 2a and 2b. First, negative sentences resulted in greater volume and higher intensity of activation than affirmative sentences in the left hemisphere, $F(1,8)=5.68, P<0.05$, for voxel counts, and $F(1,8)=5.1, P<0.05$, for intensity. This effect was due primarily to the added difficulty of negation in English, but not in J apanese, resulting in a significant interaction of polarity by language for voxel count, $\mathrm{F}(1,8)=5.95, \mathrm{P}<0.04$, and for intensity, $F(1,8)=14.67, P<0.005$. Additional tests of simple main effects of polarity indicated a significant effect for English, for voxel count, $F(1,8)=$ 6.19, $\mathrm{P}<0.04$, and for intensity, $\mathrm{F}(1,8)=8.18, \mathrm{P}<$ 0.02 , but not for $\mathrm{J}$ apanese, $\mathrm{F}(1,8)=2.05$ and $\mathrm{F}(1,8)=$ 1.2 , respectively. This interaction is compatible with the error rate data, reported below, that also showed an increase due to negation for English. Although negative sentences are behaviorally more difficult than affirmative in each language, it is only in the context of $L 2$, which imposes considerable challenges for these participants, that negation is correlated with an increase in cortical activation.

A second finding, evident in Fig. 2a, was that the total volume of activation was greater in English than in J apanese, a difference between the two languages that was reliable in the left hemisphere,
$F(1,8)=5.83, P<0.04$ for voxel count, although the average intensity measures did not statistically differ between two languages, $F<1$. This greater activation volume for English sentences over J apanese may be attributable to the difficulty of processing in a second language.

Finally, there was less activation in the right hemisphere than in the left for both English and J apanese, and the effects of language and polarity were less robust. As Fig. 2b shows, for the right hemisphere, the English conditions elicited more activation than the J apanese conditions; however, the difference was not statistically significant for the voxel count, $\mathrm{F}(1,8)=3.36, \mathrm{MS}_{\text {lang. } \times \text { subj. }}=625.76, \mathrm{P}<$ 0.1 , nor for the intensity, $F(1,8)<1$. The slight increase of activation amount by negation for English resulted in a interaction between polarity and language that was marginally significant for voxel count, $F(1,8)=4.01, P<0.08$, and significant for intensity, $F(1,8)=9.1, P<0.02$. In sum, the right hemisphere is less sensitive to the linguistic variables, although English did elicit more activation than J apanese, albeit not reliably more. The following sections focus on the left-hemisphere ROIs because the activation was more sparse in the right and because there was no statistically significant 

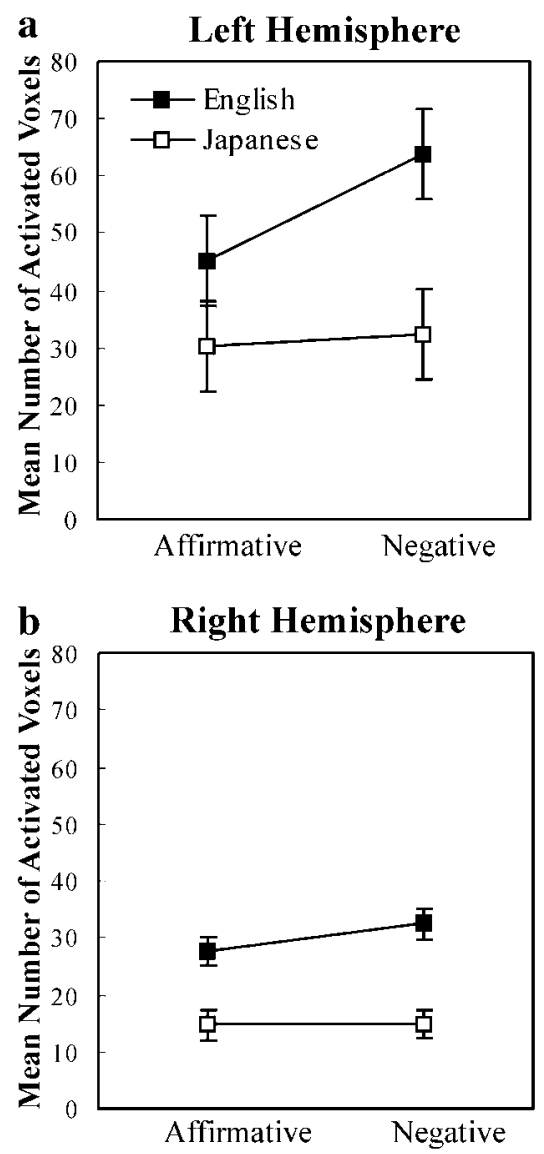

FIG. 2. The average amount of activation in terms of number of activated voxels in the (a) left and (b) right hemispheres is plotted as a function of polarity and language. The magnitude of the cortical activation increased as a function of polarity for English in the left hemisphere. The effect of language is similar in both hemispheres, though the impact was stronger in the left. In this and the following figures, the error bars represent 95\% confidence intervals based on the pooled MSefrom the corresponding language by polarity ANOVA for the appropriate region (Loftus and Mason, 1994).

language effect nor interaction by polarity for any right-hemisphere ROIs for voxel count.

\section{Classical Language Areas ${ }^{5}$}

The left temporal region has been implicated in several studies of sentence comprehension in both the auditory and the visual modalities ( $\mathrm{J}$ ust et al., 1996; Keller et al., 2001; Michael et al., 2001), and as expected, this region showed considerable activation

\footnotetext{
${ }^{5}$ The Talairach coordinates $(x, y, z)$ of the means of the activation centroids for the English negative condition and the J apanese negative condition in the classical language regions are $(-53,22,5)$ and $(-54,21,4)$ in the left temporal, $(-46,-18,19)$ and $(-46,-17,19)$ in the left pars opercularis, and $(-52,43,25)$ and $(-53,43,26)$ in the left angular gyrus, respectively. The means were based on data from participants who had activation in at least three voxels, resulting in nine, six, and five observations for the left temporal, the left pars opercularis, and the left angular gyrus, respectively.
}

for both the English and the J apanese conditions. Also, as Fig. 3a shows, this region's activation pattern mirrors that obtained for the left hemisphere averaged across ROIs. Considerably more voxels were activated (marginally reliable) and a significantly greater percentage increase in intensity occurred for English compared to J apanese $(F(1,8)=$ $4.2, \mathrm{P}<0.07$, for voxel count, and $\mathrm{F}(1,8)=5.68, \mathrm{P}<$ 0.04 , for intensity). This effect is consistent with the greater difficulty that these participants had in processing English as a second language. Also, there was a significantly greater amount of activation for negative than for affirmative sentences $(F(1,8)=$ 10.41, $\mathrm{P}<0.01$, for voxel count, and $\mathrm{F}(1,8)=7.19$, $P<0.03$, for intensity). However, only English showed significantly increased activation as a function of polarity, resulting in a reliable interaction of language by polarity for both the number of activated voxels $(F(1,8)=10.75, P<0.01)$ and their intensity $(F(1,8)=7.24, P<0.03)$, suggesting that the processing of negation in English is more demanding than in J apanese for these participants. Tests of the simple main effects of negation indicated that both measures, voxel count and intensity increase, were higher for negative than for affirmative conditions for English, $\mathrm{F}(1,8)=10.92, \mathrm{P}<0.01$ and $\mathrm{F}(1,8)=10.68, \mathrm{P}<0.01$, respectively, but neither measure showed a significant effect for J apanese, both $F(1,8)<1$. Although one cannot tell from the $\mathrm{fMRI}$ data what precise set of processes underlies the temporal region activation, this region's sensitivity to linguistic difficulty of both types is consistent with the hypotheses discussed in the Introduction, namely that the area plays a role in activating and perhaps coordinating the systems that support linguistic interpretation.

The left opercular region, the posterior part of Broca's area, has been implicated in numerous sentence comprehension studies and showed consistent activation across participants in the present study as well. However, as Fig. 3b shows, the average amount of activation was much less than for the temporal region and was not reliably modulated by negation, $\mathrm{F}(1,8)=$ 1.36 , nor by language, $\mathrm{F}(1,8)<1$, nor was there an interaction between language and polarity, $\mathrm{F}(1,8)<1$. There was very little activation in any condition in the left triangular region, the anterior part of Broca's area.

The left angular gyrus, which has been implicated in studies of verbal working memory (e.g., Awh et al., 1996), also showed activation across participants; although the effects of language and polarity were in the same direction as in the left temporal region, as shown in Fig. 3c; they were not reliable, $F(1,8)=3.18$ and $F(1,6)<1$, for the voxel count and the intensity, respectively. 

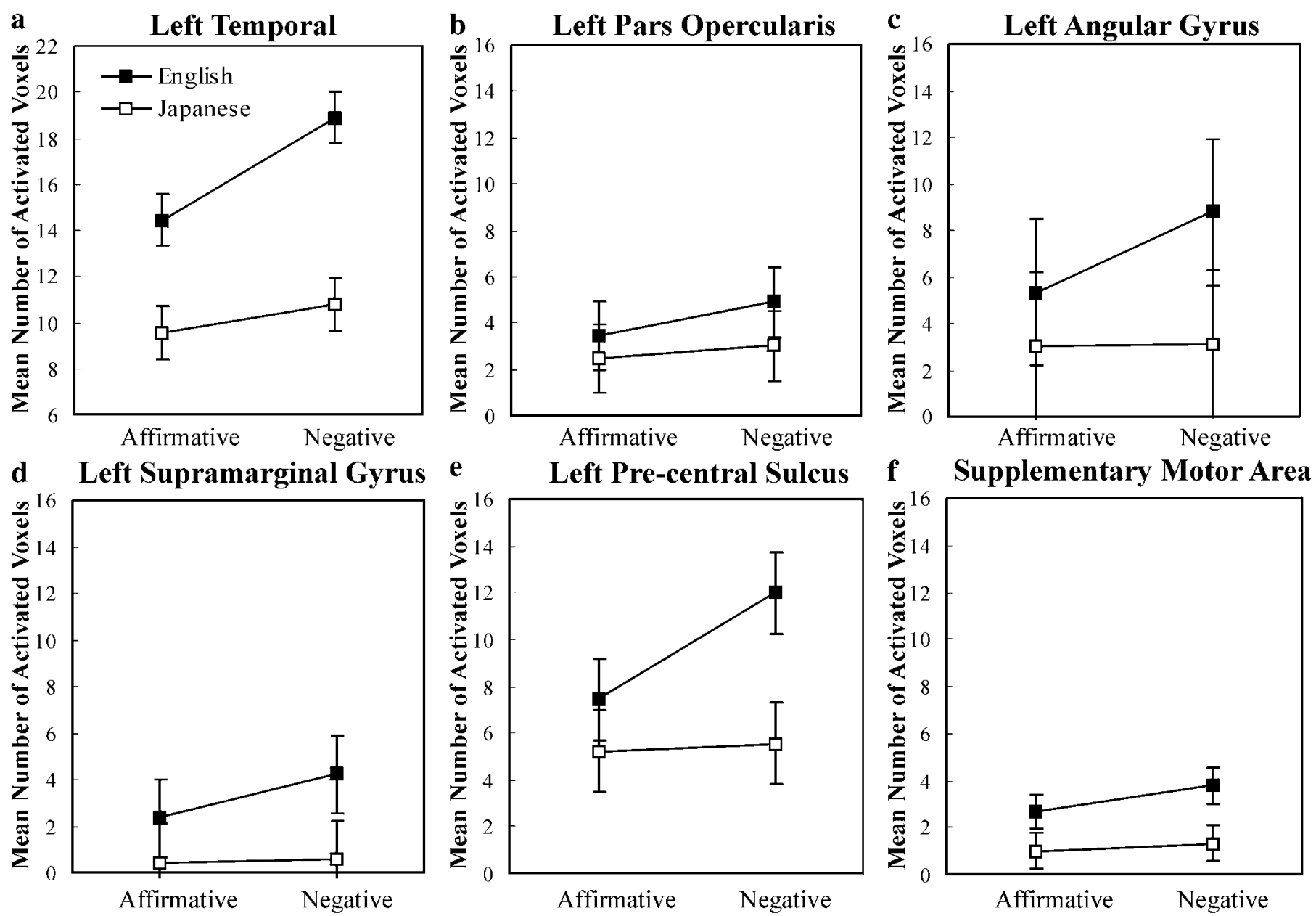

FIG. 3. The amount of activation in terms of number of activated voxels in the (a) left temporal, (b) left pars opercularis, (c) left angular gyrus, (d) left supramarginal gyrus, (e) left precentral sulcus, and (f) supplementary motor area is plotted as a function of polarity and language. All ROIs show a pattern similar to the left hemisphere, and it is most pronounced in the left temporal and left precentral sulcus. Note that the scale range of the graph is 6-22 instead of 0-16 in the left temporal region.

\section{Left Supramarginal Gyrus}

This region showed activation (see Fig. 3d), particularly in the English condition, with a mean of 3.3 voxels, with very little activation for J apanese, with a mean of 0.5 voxels, a difference that was significant for the voxel count measure $(\mathrm{F}(1,8)=7.45, \mathrm{P}<0.01)$.

\section{Left Precentral Sulcus Region}

As mentioned in the Introduction, activation in this region is typically associated with spatial attention and eye movements (e.g., Luna et al., 1998). Although eye movement responses may be sufficient to activate this area, they are apparently not necessary. Several studies (Binder et al., 1997; Michael et al., 2001) have found left-lateralized activation in this region associated with auditory sentence comprehension. This region showed considerable activation in the current listening study. What is more interesting is that the pattern across the four conditions was similar to that shown in the left temporal region. For the number of activated voxels, English elicited significantly more activation than J apanese $(F(1,8)=7.29, P<0.05$, and $F<1$ for the voxel count and intensity measures, respectively). Also negative sentences elicited more activation than affirmative ones $(F(1,8)=6.00, P<0.05$, and $F(1,8)=1.11$ for the voxel count and intensity measures, respectively), and there was a significant interaction between language and polarity $(\mathrm{F}(1,8)=7.94, \mathrm{P}<0.05$, and $F(1,8)=3.22, P<0.11$, for the voxel count and intensity, respectively). Additional tests of the main effects showed that the negation effect was significant for English, $\mathrm{F}(1,8)=8.02, \mathrm{P}<0.01$, but not for J apanese, $F(1,8)<1$; the percentage of increase in signal intensity showed a similar effect, although not statistically significant. Thus, this region shows the same effect of polarity and language as the left tem- 

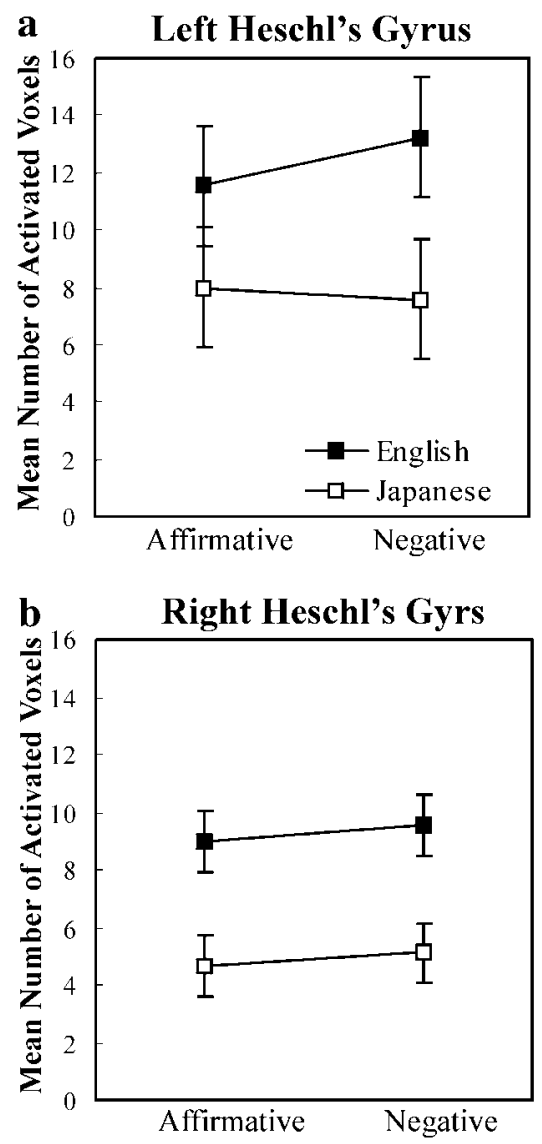

FIG. 4. The average amount of activation in terms of number of activated voxels in the (a) left Heschl's gyrus and (b) right Heschl's gyrus is plotted as a function of polarity and language. The number of activated voxels is greater for English processing than for J apanese processing.

poral region, suggesting a functional connection between the two regions.

\section{Supplementary Motor Area}

Activation of this region has been reported in studies of the short-term retention of verbal stimuli (Fiez et al., 1996; Paulesu, 1993; Petrides, 1993), suggesting that phonological rehearsal is one of the multiple functions of the supplementary motor area. Although participants showed consistent activation in this region, the magnitude was small. Nevertheless, English elicited significantly more activation than J apanese, $F(1,8)=$ $6.44, \mathrm{P}<0.05$, for the voxel count. However, the activation intensity failed to reach significance for the five participants who showed activation for both language conditions, $F(1,4)<1$. Neither the effect of negation nor its interaction with language was significant.

\section{Auditory Sensory Region (Heschl's Gyri)}

Figures $4 a$ and $4 b$ show that bilateral Heschl's gyri, the auditory sensory areas, also showed more activa- tion for the English than for the J apanese sentences, an effect that was marginally significant in the left hemisphere, $\mathrm{F}(1,8)=4.97, \mathrm{P}<0.06$ for the voxel count, but not significant in the right $F(1,8)=2.55, P<0.15$. One possible interpretation of this effect is that $E n-$ glish is more difficult to process than J apanese for these participants and so elicits more activation. Such an interpretation would suggest that even primary sensory areas reflect differences in cognitive workload and not simply sensory qualities.

\section{Behavioral Measures}

As Figure 5 shows, negative sentences took longer to verify than affirmative sentences for both English and J apanese, $F(1,9)=19.3, P<0.002$, indicating that negation contributed to the sentence comprehension difficulty. Also, the response times did not show a reliable difference between English and Japanese, $F(1,9)=1.77$, nor was there an interaction with negation, $F(1,9)=1.42$. However, participants showed a significant increase in the number of the errors for negative sentences for only English, resulting in a reliable interaction of language and polarity, $F(1,9)=$ 18.64, $\mathrm{P}<0.002$. These behavioral measures support the overall findings for the $\mathrm{fMRI}$-measured activation that the negation handling is more difficult for these participants when they are processing English than when they are processing J apanese.

The error rates varied considerably among participants, and the rates were strongly related to the amount of activation in both the English and the J apanese conditions, as shown in Fig. 6. J apanese participants who made fewer errors had more activation in the left temporal region than those who made many errors in both English $(r(7)=-0.73, \mathrm{P}<0.05)$ and J apanese $(r(7)=-0.60, P<0.10)$; the correlation between errors and activation in the English condition

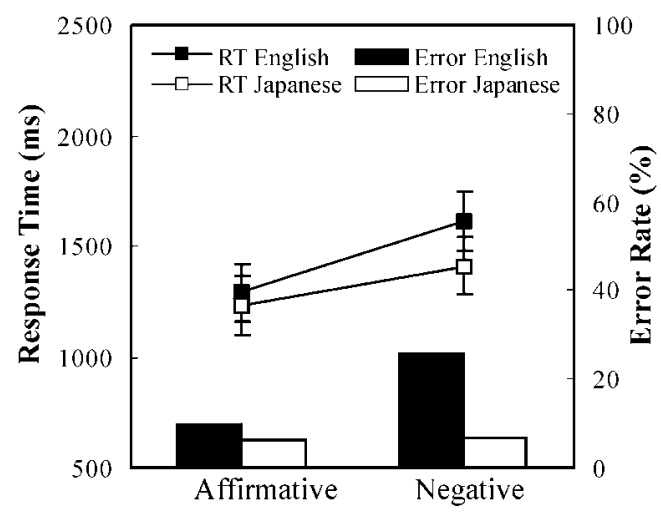

FIG. 5. Response time (lines) and error rates (bars) for the probes plotted as a function of polarity and language. Both English and $\mathrm{J}$ apanese showed increase of response time by negation. English also showed significant increase of error rate by negation, whereas J apanese did not. 

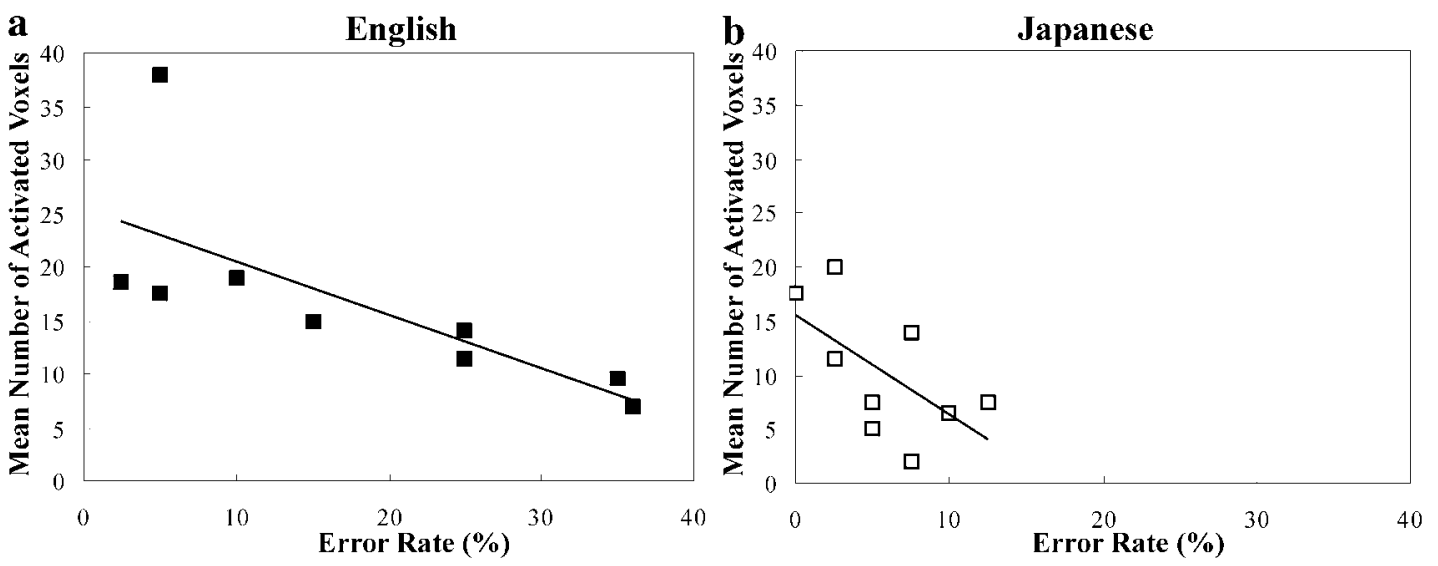

FIG. 6. The relation between comprehension level (as measured by error rate) and activation volume in the left temporal region for (a) English and (b) J apanese. The best-fitting regression lines ((a) y $=25.48-50.02 x$; (b) y $=15.48-91 x)$ indicate that the error rate is negatively related to the activation amount for English ( $r=-0.73)$, and for $\mathrm{J}$ apanese $(r=-0.60)$, although the latter correlation failed to reach significance.

persisted when the participants' self-ratings of fluency were covaried out, $(r(7)=-0.70, P<0.05)$, suggesting that the correlation is not a reflection of English Ianguage skill. This same negative relation was found for the overall amount of activation in the left and right hemispheres, although the relations were not as strong, $r(7)=-0.55$ and -0.53 , respectively. The negative correlations suggest that individuals who made more of an effort to understand the sentences and respond to the probes correctly had more activation than those who were less accurate, although for any correlation, the direction and nature of the causal relations are unclear. Nevertheless, these data suggest that comparing the amount of activation between two language conditions requires consideration of the relative error rates.

\section{GENERAL DISCUSSION}

In sum, this study suggests that there is considerable overlap in the cortical substrate that supports the processing of auditory English sentences by these native J apanese, who have acquired moderate fluency, albeit relatively late. In this way, it is in agreement with studies of highly fluent bilinguals, particularly in comprehension studies that were reviewed in the Introduction. An additional contribution has been to show that it is useful to quantify the amount of overlap and the amount of activation and that such quantification provides insights concerning the interpretation of the activation. Specifically, the additional activation associated with English in multiple cortical areas appears to be related to the processing difficulty experienced by these J apanese listeners who have no control over the rate at which they must process the spoken sentences. The argument for this interpretation comes from studies, cited in the Introduction, showing that large computational difficulty in $L 1$ for monolinguals is often associated with greater activation in a network of regions that are associated with the processing. Thus, the greater cortical recruitment for $L 2$ may be at least partially a reflection of its greater demands, rather than reflecting cortical areas that are specifically dedicated to L2, although this possibility is not precluded.

The workload perspective that motivated this study may also help reconcile the different results reported by Dehaene et al. (1997) in the Introduction. They reported less activation and higher intersubject variability for $L 2$ than for $L 1$ in the left temporal region, which they explained as due to a failure of $L 2$ to recruit the left temporal region consistently. Similarly, Perani et al. (1998) reported a smaller extent of PET-measured activation in the left temporal region for $L 2$ for lower fluency bilinguals in an auditory comprehension study. A somewhat different interpretation is suggested by the workload perspective and the current finding that the activation volume in the left temporal region correlates negatively with error rate. Specifically, in the earlier studies, activation in some cortical areas could be lower or less consistent if some participants had a lower comprehension level for L 2 compared to $L 1$. According to this account, the amount of activation reflects not only the task demand, but also the attempt on the comprehender's side. Thus, less activation does not always mean that the task is easy for the individual, but could mean that s/he is not attempting to meet the required demand. These results indicate the advantage of monitoring comprehension with behavioral measures because the activation volume is partially influenced by the degree to which the participant successfully meets the task demands.

Although English elicited greater activation than J apanese in the left temporal region, the posterior classical language region, the results also showed a 
strong and consistent language effect on verbal working memory subsystem regions, the left supramarginal gyrus, the left precentral sulcus, and the supplementary motor area. This result may indicate greater involvement of phonological rehearsal or retention for $L 2$ for moderately fluent bilinguals. Such an interpretation is also consistent with the conclusions of a behavioral study of bilinguals performing a reading span task, which requires participants to read aloud successive sentences and to recall target words at certain points (Osaka et al., 2000). Participants made more semantic errors in their native J apanese language and more phonological errors in their second language, Hungarian. Osaka et al. suggested that the participants could accomplish semantic access in parallel to the visual encoding in their native language because such access was automatic, whereas in their second language, they retained the words in the form of phonological representation, relying more on phonological recoding and rehearsal. Phonological processes may also play a role in the activation we observed in the precentral sulcus region. Although this region is also associated with spatial attention or eye movements (Kim et al., 1999; Luna et al. 1998), in our lab it has been activated in auditory sentence comprehension tasks (Michael et al., 2001), as well as in the present study, in which eye movements are unlikely to be a source of involvement.

The auditory sensory regions, Heschl's gyri, also showed higher activation for English, suggesting more processing difficulty than in J apanese. One possible account is that this region's activation shows some feedback effects, such that it is modulated by the additional workload of other regions in processing English, such as linguistic comprehension complexity or additional phonological retention. However, although there was a language effect, there was no negation effect on the activation of this region, indicating that if there is feedback, it is selective. Alternatively, or in addition, the greater activation for English may reflect the difficulty of nonnative phoneme perception. Further research is needed to determine the degree to which this area is sensitive to processing complexity and stimulus qualities of spoken language.

One interesting aspect of the workload perspective arises from the interpretation of the overadditive interaction, in which negation had more of an effect on the volume of activation in English than in J apanese. This occurred not only in the left temporal region, but also in the precentral sulcus region. The overadditive interaction indicates that the volume of activation involved in processing negative English sentences cannot be predicted from the simple sum of the effects of the two variables. Interactions of a comparable form between two workload variables have been observed in several other fMRI studies of sentence processing. For example, when both the lexical and the syntactic pro- cessing in the comprehension of a sentence were made more demanding (Keller et al., 2001), reliably more activation was observed, whereas neither variable alone produced a reliable effect. Similar results have been found in the spatial domain as well (Diwadkar et al., 2001). Overadditive interactions that arise from the presence of two or more sources of computational demand mean that the activation of some cortical areas is not predictable by considering an individual attribute of the processing. If such results turn out to be general, they suggest that conclusions concerning the location and amount of activation engendered by a given process are limited if they are based on studies that examine variation along only a single dimension that affects that process, as most neuroimaging studies do; other areas of cortical activation may be detectable only if other dimensions of complexity covary.

To summarize, the different activation patterns for $L 1$ and L 2 may partly reflect the workload involved in processing each language. Furthermore, L1 and L2 differences affect multiple levels of comprehension, including stimulus encoding and phonological retention. Because L2 imposes a greater workload on these various processes, this may influence the activation volume in the most strongly associated regions, as well as feedback among regions, and the final activation pattern of the network systems emerges from their interaction. The results more generally suggest that it may not be wise to think of a single cortical network as supporting a language, neither L1 nor L2, not even at a fixed phase of acquisition. Rather, the systems are characterized by a dynamic quality that is sensitive to the task demands and reflects the ability, within limits, to adapt through recruiting nearby cortical resources.

\section{ACKNO WLEDGMENTS}

This research was partially supported by National Institute of Mental Health Senior Scientist Awards, $\mathrm{MH}-00661$ and $\mathrm{MH}-00662$; National Institute of Mental Health Grant MH-29617; and National Institute of Neurological Disorders and Stroke Grant PO1-NS35949. Portions of this work were reported at the 64th Conference of the J apanese Psychology Association, Kyoto, J apan, November 2000. We thank Allan J uffs for his helpful discussions and his assistance in finding participants, Rob Mason and Tim Keller for their advice and help in data analysis, Yoshishige Hasegawa for his help in data processing, and Kurt Schimmel for his help in data presentation. We also thank Alice McEleney, Hideya Koshino, Holly Zajac, Natasha Tokowics, and Sharlene Newman for their helpful comments on early versions of the manuscript.

\section{REFERENCES}

Awh, E., J onides, J ., Smith, E. E., Schumacher, E. H., Koeppe, R., and Katz, S. 1996. Dissociation of storage and rehearsal in verbal working memory: Evidence from PET. Psychol. Sci. 7: 25-31.

Bandettini, P. A., Wong, E. C., Hinks, R. S., Tokofsky, R. S., and Hyde, J. S. 1992. Time course EPI of human brain function during task activation. Magn. Reson. Med. 25: 390-397. 
Binder, J . R., Frost, J . A., Hammeke, T. A., Cox, R. W., Rao, S. M., and Prieto, T. 1997. Human brain language areas identified by functional magnetic resonance imaging. J . Neurosci. 17: 353-362.

Braver, T., Cohen, J. D., J onides, J ., Smith, E. E., and Noll, D. C. 1997. A parametric study of prefrontal cortex involvement in human working memory. Neurol mage 5: 49- 62.

Carpenter, P. A., and J ust, M. A. 1975. Sentence comprehension: A psycholinguistic processing model of verification. Psychol. Rev. 82: 45-73.

Carpenter, P. A., J ust, M. A., Keller, T., Eddy, W. F., and Thulborn, K. R. 1999a. Graded functional activation in the visuospatial system with the amount of task demand. J . Cognit. Neurosci. 11: $9-24$.

Carpenter, P. A., J ust, M. A., Keller, T., Eddy, W. F., and Thulborn, K. R. 1999b. Time course of fMRI-activation in language and spatial networks during sentence comprehension. Neurol mage 10: 216-224.

Caviness, V. S., J r., Meyer, J ., Makris, N., and Kennedy, D. N. 1996. MRI-based topographic parcellation of human neocortex: An anatomically specified method with estimate of reliability. J . Cognit. Neurosci. 8: 566-587.

Chase, W. G., and Clark, H. H. 1972. Mental operations in the comparison of sentences and pictures. In Cognition in Learning and Memory (L. Gregg, Ed.). Wiley, New York.

Chee, M., Caplan, D., Soon, C. S., Sriran, N., Tan, E., Thiel, T., and Weekes, B. 1999a. Processing of visually presented sentences in Mandarin and English studied with fMRI. Neuron 23: 127-137.

Chee, M., Tan, E., and Thiel, T. 1999b. Mandarin and English word processing studied with functional magnetic resonance imaging. J . Neurosci. 19: 3050-3056.

Chee, M., Hon, N., Lee, H. L., and Soon, C. S. 2000. Relative Ianguage proficiency modulates BOLD signal change when bilinguals perform semantic judgments. Neurol mage 13: 1155-1163.

Clark, H. H., and Clark, W. G. 1972. On the process of comparing sentences against pictures. Cognit. Psychol. 3: 472-517.

Crystal, D., Ed. 1998. The CambridgeEncyclopedia of Language, 2nd ed. Cambridge Univ. Press, Cambridge, UK.

Dahaene, S., Dupoux, E., Mehler, J ., Cohen, L., Paulesu, E., Perani, D., Moortele, P., Lehericy, S., and Bihan, D. 1997. Anatomical variability in the cortical representation of first and second language. NeuroReport 8: 3809-3815.

Daneman, M., and Carpenter, P. A. 1980. Individual differences in working memory and reading. J . Verbal Learning Verbal Behav. 19: $450-466$.

Eddy, W. F., Fitzgerald, M., Genovese, C. R., Mockus, A., and Noll, D. C. 1996. Functional imaging analysis software-Computational olio. In Proceedings in Computational Statistics, pp. 39-49. Physica-Verlag, Heidelberg.

Fiez, J . A., Raife, E. A, Balota, D. A., Schwarz, J. P., Raichle, M. E., and Petersen, S. E. 1996. A positron emission tomography study of the short-term maintenance of verbal information. J . Neurosci. 16: 808- 822.

Grasby, P. M., Frith, C. D., Friston, K., Simpson, J ., Fletcher, P. C., Frackowiak, R. S. J ., and Dolan, R. J . 1994. A graded task approach to the functional mapping of brain areas implicated in auditory-verbal memory. Brain 117: 1271-1282.

Hernandez, A. E., Martinez, A., and Kohnert, K. 2000. In search of the language switch: An fMRI study of picture naming in SpanishEnglish bilinguals. Brain Lang. 73: 421- 431.

Hernandez, A. E., Dapretto, M., Mazziotta, J ., and Bookheimer, S. 2001. Language switching and language representation in Spanish-English bilinguals: An fMRI study. Neurol mage 14: 510-520.

Illes, J ., Francis, W. S., Desmond, J. E., Gabrieli, J. D. E., Glover, G. H., Poldrack, R., Lee, C. J ., and Wagner, A. D. 1999. Convergent cortical representation of semantic processing in bilinguals. Brain Lang. 70: 347-363.

J ust, M. A., Carpenter, P. A., Keller, T. A., Eddy, W. F., and Thulborn, K. R. 1996. Brain activation modulated by sentence comprehension. Science 274: 114-116.

Keller, T. A., Carpenter, P. A., and J ust, M. A. 2001. The neural bases of sentence comprehension: An fMRI examination of syntactic and lexical processing. Cereb. Cortex 11: 223-237.

Kim, K. H. S., Relkin, N. R., Lee K.-M., and Hirsch, J . 1997. Distinct cortical areas associated with native and second languages. Nature 388: 171-174.

Klein, D., Milner, B., Zatorre, R. J ., Meyer, E., and Evans, A. C. 1995. The neural substrates underlying word generation: $A$ bilingual functional-imaging study. Proc. Natl. Acad. Sci. USA 92: 28992903.

Klein, D., Milner, B., Zatorre, R. J ., Zhao, V., and Nikelski, J . 1999. Cerebral organization in bilinguals: A PET study of ChineseEnglish verb generation. NeuroReport 10: 2841-2846.

Klein, D., Zatorre, R. J ., Milner, B., Meyer, E., and Evans, A. C. 1994. Left putaminal activation when speaking a second language: Evidence from PET. NeuroReport 5: 2295-2297.

Loftus, G. R., and Mason, M. E. J . 1994. Using confidence intervals in within-subject designs. Psychonomic Bull. Rev. 1: 476- 490.

Luna, B., Thulborn, K. R., Strojwas, M. H., McCurtain, B. J ., Berman, R. A., Genovese, C. R., and Sweeney, J. A. 1998. Dorsal cortical regions subserving visually guided saccades in humans: An fMRI study. Cereb. Cortex 8: 40-47.

Michael, E. B., Keller, T. A., Carpenter, P. A., and J ust, M. A. 2001. $\mathrm{fMRI}$ investigation of sentence comprehension by eye and by ear: Modality fingerprints on cognitive processes. Hum. Brain Mapping 13: $239-252$.

Nakai, T., Matsuo, K., Kato, C., Matsuzawa, M., Okada, T., Glover, C. H., Moriya, T., and Inui, T. 1999. A functional magnetic resonance imaging study of listening comprehension of languages in human at 3-tesla comprehension level and activation of the language areas. Neurosci. Lett. 263: 33-36.

Ogawa, S., Lee, T. M., Nayak, A. S., and Glynn, P. 1990. Oxygenation-sensitive contrast in magnetic resonance image of rodent brain at high magnetic fields. Magn. Reson. Med. 14: 68-78.

Osaka, M., and Nishizaki, Y. 2000. [Characteristics of the process of the working memory in the central executive system]. In Brain and Working Memory (N. Osaka, Ed.), pp. 203-223. Kyoto Univ. Press, Kyoto.

Paraids, M. 1997. The cognitive neuropsychology of bilingualism. In Tutorials in Bilingualism: Psycholinguistic Perspectives (M. M. de Groot and J . F. Krol, Eds.), pp. 331-354. Erlbaum, Hillsdale, NJ .

Perani, D., Dehaene, S., Grassi, F., Cohen, L., Cappa, S. F., Dupoux, E., Fazio, F., and Mehler, J . 1996. Brain processing of native and foreign languages. NeuroReport 7: 2439-2444.

Perani, D., Paulesu, E., Galles, N. S., Dupoux, E., Dehaene, S., Bettinardi, V., Cappa, S. F., Fazio, F., and Mehler, J . 1998. The bilingual brain: Proficiency and age of acquisition of the second language. Brain 121: 1841-1852.

Price, C., Wise, R., Ramsay, S., Friston, K., Howard, D. Patterson, K., and Frackowiak, R. 1992. Regional response differences within the human auditory cortex when listening to words. Neurosci. Lett. 146: 179-182.

Rademacher, J ., Galaburda, A. M., Kennedy, D. N., Filipek, P. A., and Caviness, V. S., J r. 1992. Human cerebral cortex: Localization, parcellation, and morphometry with magnetic resonance imaging. J. Cognit. Neurosci. 4: 352-374.

Rypma, B., Prabhakaran, V., Desmond, J. E., Glover, G. H., and Gabrieli, J. D. E. 1999. Load-dependent roles of frontal brain regions in the maintenance of working memory. Neurol mage 9: 216-226. 
Scholosser, M. J ., Aoyagi, N., Fulbright, R. K., Gore, J . C., and McCarthy, G. 1998. Functional MRI studies of auditory comprehension. Hum. Brain Mapping 6: 1-13.

Smith, E. E., and J onides, J . 1998a. Neuroimaging analyses of human working memory. Proc. Natl. Acad. Sci. USA 95: 1206112068.
Smith, E. E., J onides, J ., Marshuetz, C., and Koeppe, R. A. 1998b. Components of verbal working memory: Evidence from neuroimaging. Proc. Natl. Acad. Sci. USA 95: 876- 882.

Vaid, J . 1983. Bilingualism and brain Iateralization. In Language Function with Brain Organization (S. Segalowitz, Ed.), pp. 315339. Academic Press, New York. 